Cahiers $d u$ MONDE RUSSE

\section{Cahiers du monde russe}

Russie - Empire russe - Union soviétique et États indépendants

$43 / 4 \mid 2002$

Intellectuels et intelligentsia

\title{
Bildungsqualifikation und bÜrgerliche Gesellschaft
}

Vergleichende Anmerkungen zu ihrer Entwicklung im ausgehenden Zarenreich

\section{Manfred HILDERMEIER}

\section{OpenEdition}

\section{Journals}

Édition électronique

URL : https://journals.openedition.org/monderusse/8520

DOI : 10.4000/monderusse. 8520

ISSN : $1777-5388$

Éditeur

Éditions de l'EHESS

Édition imprimée

Date de publication : 30 décembre 2002

Pagination : 591-600

ISBN : 2-7132-1796-2

ISSN : $1252-6576$

Référence électronique

Manfred HILDERMEIER, "Bildungsqualifikation und bürgerliche Gesellschaft », Cahiers du monde russe [En ligne], 43/4 | 2002, mis en ligne le 01 janvier 2007, consulté le 03 septembre 2022. URL http://journals.openedition.org/monderusse/8520 ; DOI : https://doi.org/10.4000/monderusse.8520 
chercher : repérer : avancer

Cet article est disponible en ligne à l'adresse :

http://www.cairn.info/article.php?ID REVUE=CMR\&ID NUMPUBLIE=CMR 434\&ID ARTICLE=CMR 4340591

\title{
Bildungsqualifikation und bÜrgerliche Gesellschaft. Vergleichende Anmerkungen zu ihrer Entwicklung im ausgehenden Zarenreich
}

\section{par Manfred HILDERMEIER}

\section{Editions de l'EHESS | Cahiers du monde russe}

\author{
$2002 / 4$ - Vol 43 \\ ISSN 1252-6576 | ISBN 2713217962 | pages 591 à 600
}

Pour citer cet article :

-HILDERMEIER M., Bildungsqualifikation und bÜrgerliche Gesellschaft. Vergleichende Anmerkungen zu ihrer Entwicklung im ausgehenden Zarenreich, Cahiers du monde russe 2002/ 4, Vol 43, p. 591-600.

Distribution électronique Cairn pour les Editions de l'EHESS.

(C) Editions de l'EHESS. Tous droits réservés pour tous pays.

La reproduction ou représentation de cet article, notamment par photocopie, n'est autorisée que dans les limites des conditions générales d'utilisation du site ou, le cas échéant, des conditions générales de la licence souscrite par votre établissement. Toute autre reproduction ou représentation, en tout ou partie, sous quelque forme et de quelque manière que ce soit, est interdite sauf accord préalable et écrit de l'éditeur, en dehors des cas prévus par la législation en vigueur en France. Il est précisé que son stockage dans une base de données est également interdit. 


\section{BILDUNGSQUALIFIKATION UND BÜRGERLICHE GESELLSCHAFT}

\section{Vergleichende Anmerkungen zu ihrer Entwicklung im ausgehenden Zarenreich}

Das Thema meines Vortrags ist nicht neu. Es gehört zu den ,klassischen“ Fragestellungen der Entstehung politischer Systeme. Weder der Liberalismus noch die nationale Bewegung noch die Demokratie als selbstregulierte, in diesem Sinne kybernetische Ordnung sind ohne die Verbreitung von Bildung denkbar. Zur modernen Demokratie gehörte daher immer auch die Existenz einer sozialen Schicht, deren Kennzeichen und Definitionsmerkmal der Besitz einer besonderen Qualifikation war. Allerdings waren und sind die Begriffe für diese Schicht und die Rolle, die sie im politischen Prozeß übernahm, sehr verschiedenartig. Darin spiegelt sich zu einem erheblichen Teil die höchst unterschiedliche soziale und politische Entwicklung in den einzelnen Ländern wider, verbunden mit dem unterschiedlichen Gewicht und Charakter des Staates.

Einige der Begriffe und Konzepte, die mit ihnen oft verbunden waren, seien hier kurz genannt. Sie bilden sozusagen den Vorrat, aus dem Überlegungen für die russische Entwicklung um die Wende zum vergangenen Jahrhundert zu gewinnen sind. Danach sollen provisorische Bemerkungen die Strategie verdeutlichen und die Untersuchungsfelder abstecken, die in meiner Sicht den größten Erfolg versprechen.

Im mitteleuropäischen Raum der Frühen Neuzeit hießen diejenigen, deren wichtigstes Kapital nicht der Besitz, sondern Kenntnisse und Denkvermögen waren, bekanntlich Gebildete und nicht Intellektuelle. Auch in der Rückschau wird man bei diesem Begriff bleiben wollen. Dazu gibt nicht nur ein Begriffshistorismus Anlaß, der auf terminologische Genauigkeit achtet. Auch die Sache, die bezeichnet wird, war eine andere. Zwar verdankten auch die Juristen und Kameralisten, die in den mitteleuropäischen Territorialstaaten ausgebildet wurden, ihre Funktionen und Ämter im wesentlichen ihren einschlägigen Qualifikationen. Die Universitäten, die sich etwa im 
Deutschen Reich beinahe jeder Landesherr zulegte, dienten ja eben diesem Zweck, den wachsenden Bedarf an einschlägigem Wissen zu befriedigen. Aber dieses Wissen war eben so eng mit dem Staatsdienst verbunden, daß seine Träger nur in dieser Funktion gesehen wurden, nicht als sozusagen separate Inhaber von Qualifikation.

Ähnliches gilt für die zweite große Gruppe von Gebildeten in diesen Jahrhunderten, die protestantischen Pastoren, die bekanntlich ein Universitätsstudium absolvieren mußten, oder die katholischen Pfarrer, die die Jesuitenschulen der Gegenreformation durchlaufen hatten. Und auch, als im 18. Jahrhundert erstmals ein kleiner Kreis von Personen entstand, die unabhängig vom Staatsdienst als Verleger, Dichter, Bibliothekare, Publizisten und frühe Journalisten von ihren intellektuellen Fähigkeiten lebten, nannte man sie nicht so, weil sich inzwischen das geistige Leitideal geändert hatte: An die Stelle der Ausbildung war die Bildung im Sinn der Formung der Gesamtpersönlichkeit getreten. Die Aufklärung wollte nicht mehr bloß Fachwissen und ,äußerliches' know how vermitteln - und in nichts anderem bestand die juristische und kameralistische Schulung des 17. Jahrhunderts - , sondern den Charakter der Gebildeten durch die Erweiterung ihres geistigen Horizonts verändern. Dieser Bildungsbegriff war breiter, er umfaßte mehr als das bloße Lesen, bezog die Erfahrung ein - wie die großen ,Bildungsromane' des 18. Jahrhunderts bezeugen -, beruhte aber im Kern auf Wissen. Nur war dieses Wissen stärker auf sich selbst gerichtet, sah das Ich in Verbindung mit der Umwelt und zielte auf die Veränderung beider. Bildung war (und ist) in diesem Sinne reflexiv. Nicht zuletzt darin unterscheidet sie sich von bloß technisch-fachlicher Fertigkeit (Fähigkeit) als Inhalt der Ausbildung. Streng genommen gibt es auch den ,Intellektuellen` erst seit diesem kritischen Bezug auf das Selbst und seine Umwelt im Bildungsideal der Aufklärung; bis heute unterscheidet sich der Intellektuelle vom bloßen Spezialisten dadurch, daß er sein Wissen und seinen trainierten Verstand auch auf sich selbst anwendet. Im intellektuellenfeindlichen konservativnationalistischen Diskurs der Zwischenkriegszeit formulierte man das so: Ein Intellektueller ist der, der seinem Verstand nicht gewachsen ist (Definition des „Großen Brockhaus“"von 1941). Dies war immer vor dem Hintergrund des Gegensatzes zum Spezialisten zu lesen, dessen Fertigkeiten, wie man weiß, ,neutral` waren und von autoritär-diktatorischen Regimen ebenso benötigt wie benutzt wurden .

Es liegt nun auf der Hand, daß Begriff und Erscheinung der Bildung nicht zufällig ungefähr zur selben Zeit auftauchten wie Begriff und Erscheinung der Öffentlichkeit (obščestvennost'). Beide waren inhärent miteinander verbunden. Wenn Bildung den selbstreflexiven Gebrauch der Verstandesfähigkeit zum Zwecke der Vervollkommnung meinte, schloß er bestimmte Vorstellungen über die politischen Rahmenbedingungen dieses Gebrauchs ein. Autonome Vernunft verlangte nach Selbstbestimmung und einer Freiheit, die letztlich keine individuelle bleiben konnte. Wenn sich das Individuum letztlich nur mit seinem Kontext verändern und verbessern konnte, mußte dieser Kontext auch offen für Verbesserung sein. In dieser Symbiose von privater und öffentlicher Selbstbestimmung liegt die tiefere, sachliche Ursache für die Affinität zwischen Aufklärung und einer Form der politischen Herrschaftsorganisation, die in der Demokratie oder parlame- 
narischen Monarchie ihre konsequenteste Verwirklichung fand. Weil aber die Herrschaftsorganisation im 18. und 19. Jahrhundert auf dem europäischen Kontinent mit Ausnahme des revolutionären Frankreich traditional-dynastisch war, enthielt die autonomieorientierte Öffentlichkeit zunächst ein erhebliches kritisches Potential. Dies ist die Kernidee der Habermas'schen Konstruktion der „,bürgerlichen Öffentlichkeit“" (1958) ${ }^{1}$. Öffentlichkeit ist die gleichsam politisch gewendete, das heisst entprivatisierte Version der Geselligkeit (verstanden als Kommunikation von Angesicht zu Angesicht, „Kaffeehaus-Modell“); sie zielt auf die freie und das heißt auch die herrschaftsfreie Anwendung von Vernunft und ist mithin solange kritisch-oppositionell, wie Herrschaft und der von ihr bestimmte öffentliche Raum nicht mit den Funktionsprinzipien selbstbestimmter Vernunft übereinstimmen.

Freilich: der Begriff der „,bürgerlichen Öffentlichkeit“ ist ein Idealtypus im Sinne Max Webers. In der Wirklichkeit hat es ihn so nicht gegeben. Er lebt vom Gegensatz zwischen „Gesellschaft“ und „Staat“, der etwa in England fehlte. Die public sphere war immer etwas anderes als die ,,bürgerliche Öffentlichkeit“. Ihr fehlte die emphatische Opposition gegen einen Staat, der vom vor-rationalen dynastischen ,,monarchischen Prinzip“, wie man im 19. Jahrhundert sagte, bestimmt wurde. Dies ist allerdings kein Einwand gegen die gedankliche Konstruktion selbst. Der Idealypus lebt bekanntlich per definitionem von der Überzeichnung (Betonung) charakteristischer Merkmale auf Kosten der historischen Genauigkeit. Sein heuristischer, Erkenntnis begründender Wert - und das sollten auch Historiker so sehen - kompensiert den Mangel an Detailtreue. Aber die mangelnde Übereinstimmung mit dem ,Realtypus“ sollte zu zwei anderen Einschränkungen Anlaß geben.

Zum einen müßte deutlicher gemacht werden, daß dieser Typus an eine bestimmte Phase der mitteleuropäischen, historischen Entwicklung gebunden war. Habermas hat selbst in seinem Vorwort zur Neuauflage seines oben genannten Buches im Jahre 1990 eingeräumt, daß er die Beeinflussung des öffentlichen Raumes durch die Herrschaft, von ihm als „Vermachtung“ bezeichnet, zu wenig bedacht habe. Dies gilt insbesondere für die Steuerung der öffentlichen Meinung durch die aufkommenden Massenmedien. Die Zeitpunkte, zu dem sich die Medien in den einzelnen Ländern durchsetzten, waren sicher sehr unterschiedlich. Desgleichen muß offen bleiben, in welchem Maße sie die Kritik verstärkten oder sich der Staatsmacht unterwarfen. In jedem Fall beginnt hier etwas Neues, das einer neuen begrifflich-gedanklichen Konstruktion bedarf.

Zum anderen sind neben den Ideen die Interessen stärker zu gewichten. Sicher sorgten die aufgeklärten Geister für die gedankenscharfe Formulierung ihrer Vorstellungen vom öffentlichen Vernunftgebrauch. Aber für deren Durchsetzung brauchten sie, wie eh und je, die Unterstützung vieler. Dabei mag offen bleiben, ob diese ,Vielen“ aus Überzeugung und - mit einem Ausdruck Kants - interesselosem Wohlgefallen' handelten oder auch ,lebensweltlich-materielle" Absichten damit verbanden. Den Befürwortern der Kulturgeschichte ist sicher darin zuzustimmen, daß ,immateriellen“ Idealen und Werten eine größere Bedeutung für 
Handlungen zugeschrieben werden sollte, als das häufig der Fall war. In jedem Falle macht erst die Verbindung des Ideals öffentlicher Selbstbestimmung als notwendiger Kontext selbstreflexiven Vernunftgebrauchs mit den Wünschen (materiellen wie immateriellen) einer breiteren Gesellschaftsschicht verständlich, warum sich die liberal-demokratische Bewegung im Laufe des 19. Jahrhunderts mit solcher Gewalt Bahn brach. Fundament und Kitt dieser Verbindung war in meiner Sicht der Kernprozeß der Neuzeit: die Emanzipation des Individuums, die seit dem Ende des 18. Jahrhunderts eine neue, politische Dimension erhielt. Diese wiederum hing aufs engste und untrennbar mit wachsenden Qualifikationsansprüchen an das Individuum zusammen. Je qualifizierter ein wachsender Teil der Gesellschaft wurde, desto größer war das Potential von Forderungen nach weitgehender Selbstbestimmung im öffentlich-politischen Raum - jedenfalls solange es, wie dann im 20. Jahrhundert, keinen Staat gab, der zu einem neuen Ausmaß an Gewaltanwendung und zur Massenmanipulation fähig war. Im Sinne dieser elementaren Affinität von Qualifikation, individueller Emanzipation und politischer Demokratisierung galt in der Tat, wie Lothar Gall vor 25 Jahren formulierte, daß nicht die ,bürgerliche Klassengesellschaft" als Ziel in diesem Prozeß angelegt war, sondern die ,klassenlose Bürgergesellschaft ${ }^{\star}$. Er bezog seine Dynamik aus der Utopie der Verallgemeinerung selbstbestimmter Partizipation, die nur teilweise mit begrenzten sozialen Interessen zusammenfiel ${ }^{2}$.

Auf diese Weise kann man die liberal-demokratische Verfassung einer Gesellschaft und ihres öffentlichen Raums von mindestens vier Bedingungen lösen, die bislang zumeist mit ihr verbunden wurden. Bürgergesellschaft und bürgerliche Öffentlichkeit sollten getrennt werden von

(1) einem sozialen Substrat namens Bürgertum. Dies ist inzwischen ein Gemeinplatz, der aber etwa in der russischen Geschichte noch kaum Berücksichtigung gefunden hat: Es gab Bürgerlichkeit als Sammelbegriff für bestimmte Werte, Normen, Denkgewohnheiten und Verhaltensweisen auch unabhängig von einer breiten bürgerlichen Gesellschaftsschicht. Diese Feststellung bedeutet nicht, daß es gar keines sozialen Trägers bedurfte, um entsprechende Mentalitäten zu propagieren und zu verankern. Aber sie läßt die Möglichkeit zu, daß andere Schichten diese Funktion übernahmen. Je nach den Gegebenheiten kamen dafür der Adel oder staatsnahe Beamtengruppen in Frage, in außereuropäischen Ländern womöglich auch Teile religiöskirchlicher Gruppen. Voraussetzung war eigenlich nur eine bestimmte bildungsmäßige Qualifikation, die die grundsätzliche Möglichkeit der Rezeption externer Ideen begründete. In diesem Sinn fügt sich der Begriff der Bürgerlichkeit auch in Überlegungen zu den Formen und Modalitäten der Beziehungen zwischen Staaten und Gesellschaften ein. Ein Bürgertum kann nicht transferiert werden, Bürgerlichkeit sehr wohl. Die sogenannte

2. Vgl. L. Gall, Liberalismus und „,bürgerliche Gesellschaft“. Zu Charakter und Entwicklung der liberalen Bewegung in Deutschland, Historische Zeitschrift, 220, 1975, S. 324-356, wiederabgedruckt in: Ders., Bürgertum, liberale Bewegung und Nation. Ausgewählte Aufsätze [zum 60. Geburtstag], München, 1996. 
Rückständigkeitstheorie hat diese Dimension des Austauschs kaum beachtet. Sie stand auch in dieser Hinsicht unter dem prägenden Einfluß von Alexander Gerschenkron $^{3}$, der ja bekanntlich Ökonom war. Gegenüber dieser Verengung ist daran zu erinnern, daß auch Ideen übernommen werden können, vom Kunstgeschmack bis zu politischen Vorstellungen;

(2) sollten Bürgergesellschaft und bürgerliche Öffentlichkeit ferner getrennt werden sowohl von einer expliziten Opposition gegen den Staat als auch von der Existenz eines Freiraumes, den ihnen ein gleichsam nicht vorhandener Staat eröffnen würde. Autonome Aktivitäten im Sinne von solchen, die aus eigenem Antrieb erfolgen, mußten nicht unbedingt gegen den Staat oder irgendeine Obrigkeit gerichtet sein. Sie mußten auch nicht politisch sein. Sie konnten sich im vorpolitischen Raum bewegen, deshalb auch von der Obrigkeit unbehelligt bleiben, aber doch eine andere Form der Öffentlichkeit und Geselligkeit erzeugen, als sie der idealtypischen Untertanengesellschaft eigen waren. Das galt für Radfahrvereine ebenso wie für Leseklubs und gelehrte Gesellschaften. Hier bildeten sich neue Netzwerke von Individuen verschiedener Berufe und Herkunft. Gemeinsam waren ihnen die jeweiligen Interessen, die den Vereinen und sonstigen Assoziationen zugrunde lagen. Voraussetzung war hier erneut ein bestimmtes Bildungs- und Qualifikationsniveau. Ansonsten aber fand, abstrakt gesprochen, Vergemeinschaftung nicht nach den tradierten Kriterien statt, nicht nach Ständen, Regionen, Wohnorten oder auch Konfessionen, sondern nach den jeweiligen, sehr unterschiedlichen Zwecken der neuen Vereinigungen;

(3) sollten Bürgergesellschaft und bürgerliche Öffentlichkeit trotz ihrer Verbindung mit allgemeinen Interessen mitsprachebereiter Gesellschaftsglieder von kollektiven sozialen Interessen getrennt werden. Um partikulare Interessen geltend zu machen, gab es zumindest in einer späteren Entwicklungsphase der ,Bürgergesellschaft ${ }^{\star}$ andere Organisationen, die man üblicherweise als Verbände bezeichnet. Von Börsenkomitees über Bankenvereine bis zu Industriellenverbänden hatten sich etwa in Rußland Interessenverbände gebildet, die jeweils ihre spezifischen Wünsche anmeldeten. Wie gerade die Geschichte des Zarenreichs zeigt, kamen sie durchaus ohne Öffentlichkeit, Demokratie und Parlament aus. Sie bildeten weder eine notwendige Vorform noch Teile einer sich selbst regulierenden Öffentlichkeit in einer parlamentarischen politischen Ordnung. Sie waren nur einfach etwas anderes;

(4) schließlich sollte man sich auch von der Vorstellung lösen, daß Bürgergesellschaft und bürgerliche Öffentlichkeit in jedem Fall von einer breiten Schicht im Sinne einer gewisssen Mindestgröße getragen werden müssen. Sicher haben Massenbewegungen, die auch liberale Ideen transportierten wie etwa die nationale Bewegung oder auch einschlägige Traditionen wie die stadtbürgerliche Freiheit ihre Durchsetzung gefördert. Aber es ist nicht auszuschließen,

3. Vgl. A. Gerschenkron, Economic backwardness in historical perspective, Cambridge, MA, 1962. 
wenngleich diese Überlegung eher analytisch konsequent als historisch belegt ist, daß es ausreicht, „strategische Eliten“ dafür zu gewinnen. Sie können ebenso als Saatbeet fungieren wie z. B. Alphabetisierungsgesellschaften für die Entstehung von Nationalismus. Vor allem in Gesellschaften, die in sozioökonomischer Hinsicht nicht zu den bürgerlichen zu zählen sind, können solche Eliten in diesem Sinne wirken. Dies setzt erneut eine erhebliche Offenheit für solche Ideen und sozusagen die Bereitschaft und Fähigkeit voraus, sich mit der Zukunft des Gemeinwesens zu befassen. Auch in dieser Hinsicht fällt den Gebildeten eine besondere Rolle und Aufgabe zu. Sie kann, und dies ist ein alter Gedanke, als Ersatz für ,bürgerliche' Schichten fungieren, aber nicht - wie einst Trotzki und andere Marxisten meinten —, weil sie selbst ,bürgerlich “ war, sondern weil sie am ehesten die erforderlichen geistigen Voraussetzungen und den nötigen Horizont mitbrachten.

Natürlich verfolgen solche Überlegungen nicht zuletzt den Zweck, ein Konzept von bürgerlicher Gesellschaft zu gewinnen, das auf andere Verhältnisse übertragbar ist. Komparative Begriffe und Interpretationsfiguren dieser Art müssen eine durchaus schwierige Balance zwischen Identität im Kern und Flexibilität in den Rahmenbedingungen halten. Über die Abgrenzung zwischen beiden kann man streiten; sie bedarf auch jeweils der Überprüfung. Aber wenn man den Kern, die Identität, im Ziel und Inhalt lokalisiert, kann ein und dieselbe Sache durchaus unterschiedliche Träger zu unterschiedlichen Zeiten in unterschiedlichen Kontexten haben. Kern der „bürgerlichen Gesellschaft“ in diesem Sinne wäre das, was zur Verdeutlichung der Loslösung vom 18. Jahrhundert und vom Bürgertum im sozialen Sinn inzwischen häufig ,Zivilgesellschaft“ genannt wird. Bei allem inflationären Gebrauch, den dieser Begriff in den letzten anderthalb Jahrzehnten erlebt hat, ist er doch nicht beliebig füllbar. Er bleibt ein Gegenbegriff zu autoritären Konzeptionen von politischer Ordnung und kreist um mindestens zwei Kernbestimmungen:

1. Autonomie im Sinne von Abwesenheit fremder Veranlassung und

2. Pluralismus im Sinne der Abwesenheit einer hegemonialen Kraft, sei dies der Staat, die Kirche, das Militär oder eine dominante Partei.

Zivilgesellschaft in diesem Sinn verträgt sich weder mit Autoritarismus noch mit Klerikalismus, Militarismus oder dem Monopol einer oder mehrerer Parteien. Ihr Grundprinzip ist letztlich das der Balance und Ausgewogenheit.

Nun ergibt sich diese Ausgewogenheit in einer differenzierten Gesellschaft durch die Vertretung unterschiedlicher Interessen und die Konkurrenz unterschiedlicher Ideen oder zumindest durch das Streben danach. Wo sich dieser Prozeß noch in den Anfängen befindet oder tradierte Institutionen, wie z. B. der Staat, übermächtig sind, ergeben sich Verschiebungen und Funktionsverlagerungen. Man sollte an dieser Grundidee aller Vergleiche zwischen Entwicklungen in unterschiedlichen Kontexten, in unterschiedlicher zeitlicher Erstreckung und mit unterschiedlicher Geschwindigkeit festhalten. Aber man sollte die funktionalen Äquivalente nicht als andere Entwicklungen betrachten, sondern als Varianten auf dem Wege zu einer substantiell ähnlichen politisch-sozialen Ordnung. 
In diesem Sinn hat in den letzten Jahren eine Neubewertung der vorrevolutionären Geschichte Rußlands im allgemeinen und ihrer „zivilgesellschaftlichen“ Elemente im besonderen stattgefunden. Dabei kam und kommt den im weitesten Sinn „gebildeten Schichten“ eine besondere Rolle zu. Nicht ohne Grund trägt ein einflußreicher amerikanischer Sammelband den Untertitel „Educated society and the quest for public identity in late Imperial Russia“4. Daß dieser Sammelband schon 1991 erschien, also in den Jahren zuvor vorbereitet wurde, weist im übrigen darauf hin, daß der Untergang der Sowjetunion und der Wandel der politischen Großwetterlage nur ein Anstoß unter anderen war. Zu den äußeren Motiven, von denen jede historische Forschung abhängt, kamen zumindest im Westen inneremethodische hinzu. Es war nicht zufällig die Abkehr von der Sozialgeschichte der 70er und 80er Jahre und die Hinwendung zur Kulturgeschichte, die diese Umwertung einleitete. Zwar sind manche Gegenstände dieselben geblieben, etwa die zemstva, aber die Leitperspektiven haben sich verändert. An die Stelle langfristiger Strukturen und einer unbezweifelbaren Tendenz, vom Ende her zu denken, ist die Frage nach Ansätzen, nach fragmentarischen Entwicklungen und ihrem Potential getreten. Dabei sind verschiedene Strategien gewählt und unterschiedlich weit entwickelt worden. Die meisten - und das kann kaum anders sein - haben mit verschiedenen Arten von Bildung, sei es ästhetische oder professionelle, und allesamt mit den Städten und ihrer Oberschicht zu tun. Dies kann nicht anders sein, da die meisten (aber natürlich nicht alle) Formen eigenständigen gesellschaftlichen Engagements von der Elite mit Besitz und Bildung ausgingen. Einige neuere Fragestellungen richten sich aber auch in einem engeren, spezifischen Sinn an die Rolle gebildeter Schichten im Sinne akademischer Qualifikation.

Insgesamt hat diese Umwertung nach meiner Einschätzung noch nicht sehr weit geführt. Der erwähnte Sammelband ist bei der Skizzierung jeweils neuer Fragen an zumeist bekannte Gegenstände stehengeblieben und hat noch keine Nachfolge im Sinne einer Nacharbeit gefunden. Gründlich ist eigentlich nur, nicht zuletzt von russischer Seite, das Mäzenatentum großbürgerlicher Kreise untersucht worden. Vielversprechend aber scheinen mir Untersuchungen zu sein, die im weiteren Sinn versuchen, Überlegungen aus dem Kontext der Bürgertumsforschung auf Rußland zu übertragen.

Dabei richtet sich das Interesse erstens auf eine tiefere, gleichsam subkutane Ebene und damit auch auf überwiegend nichtformalisierte Gemeinschaften im vorpolitischen Raum. Die inzwischen vielzitierten ,Netzwerke' treten an die Stelle von Organisationen; freie Vereinigungen übernehmen den ,forschungsstrategischen * Platz formalisierter Zusammenschlüsse. Überkommene, vielfach auf ,objektiven“ Kriterien beruhende Kategorien werden als zu grob und verfälschend verworfen. Statt dessen bemüht man sich, die Feinstrukturen der innerstädtischen Kommunikation freizulegen. Die Fragen richten sich weniger auf Klassen und Schichten als auf Verbindungen und Kontakte quer durch die verschiedenen Tätigkeitsbereiche von

4. Edith W. Clowes, Samuel D. Kassow, James L. West, eds, Between tsar and people. Educated society and the quest for public identity in late Imperial Russia,. Princeton, NJ, 1992. 
Subjekten und Gruppen. Vereinfacht und bildlich gesagt, verfolgt die Betrachtung statt gerader, horizontaler und kongruenter Linien unregelmäßige Formen, die von verschiedenen Kernen als eine Art von Mittelpunkten ausgehen. Da Eliten untersucht werden, bewegen sich diese Formen aber zugleich an der Spitze der städtischen Gesellschaft als ganze. Implizit operiert man daher auf der Grundlage der Annahme, daß die Interaktion zwischen diesen Gebilden eine Wirksamkeit und Ausstrahlung besitzt, die deutlich über deren eigene Grenzen hinausgehen. Insofern besteht doch eine gewisse Übereinstimmung mit der überkommenen Stratifikationsvorstellung, die aber zugleich differenziert und überwunden wird.

Die Feinstrukturen und 'Vernetzungen' lassen sich zweitens natürlich nur insoweit fassen, als sie sich formalisiert niedergeschlagen haben oder ansonsten aktenkundig geworden sind. Bloße Teegespräche und Sektfrühstücke, die es unter Kaufleuten regelmäßig gegeben haben soll, werden höchstens in Memoiren erwähnt, eignen sich aber nicht für genauere Untersuchungen. In den Mittelpunkt einiger der neuen Studien sind daher Vereine, Kunstgesellschaften, Klubs, gelehrte Gesellschaften, Bibliothekszirkel und ähnliche Einrichtungen gerückt, die gleichsam kurz vor der nicht mehr faßbaren Privatsphäre angesiedelt sind. In der klassischen, auf Hegel zurückgehenden Begrifflichkeit wären sie zwischen Individuum bzw. Familie und Staat anzusiedeln und würden zum Bereich der Öffentlichkeit zu rechnen sein. Deren Studium ist nun wahrlich nicht neu, war aber eher Gegenstand der historischen Soziologie. Auch Vereine sind spätestens in den Blick geraten, seit sie vor einem Vierteljahrhundert zu Nachfolgern der Korporationen und Stände der Gesellschaft des Ancien régime erklärt wurden (Th. Nipperdey) ${ }^{5}$. Aber auch sie sind erst in die Schlüsselfunktion eingerückt, die ihnen neuere Studien zuweisen, als die gesamten erkenntnistheoretischen Grundannahmen der Geschichtswissenschaft den Subjekten, ihrer Wahrnehmung und ihrem Handeln untereinander, wieder mehr Aufmerksamkeit widmete.

Neben institutionalisierten Formen der Kommunikation in strategisch bedeutsamen Gruppen der städtischen Elite widmen drittens neue Untersuchungen auch den nichtinstitutionalisierten Formen breiten Raum. Dies gilt in besonderem Maße, so weit sie sich von der Betonung individueller oder kollektiver Wahrnehmung und Erfahrung inspirieren lassen. Solche Studien bedürfen in besonderem Maße klarer Grenzen ihres Gegenstandes. Da die Grenzen von Institutionen und Organisationen fehlen, tritt die Stadt als annähernd definierte Größe an ihre Stelle. Sie rückt besonders für die, die mit wachen Augen sehen, zum Erfahrungs- und Handlungsraum auf. Sie wird Akteur und Adressat der Selbstbestätigung und Selbstvergewisserung in Jubiläen, Festen und Ritualen.

Der gemeinsame Bezugspunkt fast aller genannten Einzelinteressen weist viertens auf die innere Nähe der einschlägigen Untersuchungen zu politischen Organisationen insbesondere liberaler Orientierung hin. Überwiegend bewegten sich die autonomen Vergemeinschaftungen im präpolitischen Raum. Sie alle enthielten

5. T. Nipperdey, Der Verein als soziale Struktur im späten 18. und frühen 19. Jahrhundert, in Ders., Gesellschaft, Kultur, Theorie, Göttingen, 1976, S. 174-205. 
aber das Potential für eine Politisierung. Bislang bestätigt sich die Vermutung, daß es überwiegend ein- und dieselben Personen waren, die sich in Vereinen und Klubs sowie in den Stadtdumen und deren Kommissionen trafen. Insofern spielte sich Kommunalpolitik nicht nur zu einem erheblichen Teil außerhalb der dafür vorgesehenen Gremien ab. Ebenso war sie in ähnlich hohem Maße eine Angelegenheit der städtischen Oberschicht, die mehr und mehr Geschmack daran fand, ihre Probleme zumindest auf kommunaler und regionaler Ebene selbst zu lösen. Spätestens an diesem Punkt schlug die lokale Vergemeinschaftung in ein Politikum von eminenter Bedeutung um.

Schließlich ist fünftens evident, daß die potentiell politischen Vergemeinschaftungen als moderne unterstellt werden, d. h. als solche, die geburtsständische Trennschranken überwanden. Eben den Schwierigkeiten und Besonderheiten dieses Prozesses in einem Staat, der sich immer noch weitgehend auf den landbesitzenden Adel stützte, gilt sozusagen die spezifische Anpassung der aus der allgemeinen Geschichte kommenden Fragen an die russischen Bedingungen. Dabei liegt der Akzent aber nun nicht mehr auf den Hemmnissen. Vielmehr stellt sich im Maße intensiver Auswertung lokaler Materialien heraus, daß dieser entscheidende Vorgang der Herausbildung einer transständischen Elite in den Städten erheblich weiter vorangeschritten war als bislang bekannt war. Dabei kam der neuen Schicht der Gebildeten, insbesondere der akademisch Qualifizierten allem Anschein nach eine besondere Bedeutung zu. Für Odessa und Kazan' - beides nicht zufällig Universitätsstädte - hat man gezeigt, daß sich die universitäre ,Gesellschaft ‘ im weiteren Sinne in den Klubs und den informellen Zirkeln im Umkreis der lokalen Zeitungen und des Stadtrats engagierte und mit den höheren Beamten und der Bourgeoisie im klassischen Sinn des Wirtschaftsbürgertums zu dieser neuen Elite verschmolzen ${ }^{6}$.

So wie der gesamte Vorgang selbst würde auch dies zumindest eine mitteleuropäische Erfahrung widerspiegeln: Gebildete eigneten sich als Ferment einer solchen Verschmelzung am ehesten, weil sie am wenigsten eigene Interessen hatten. Zugleich bedurfte eine neue Elite dieser Art, wollte sie Einfluß ausüben einer festen Verortung in einer Stadt und einer Region sowie einer gewissen materiellen Macht. Diese fand sie in den genannten anderen Gruppen. Ideen und Interessen kamen zusammen - durchaus eine Verbindung, die nicht ohne Zukunftschancen war.

Seminar für Mittlere und Neuere Geschichte

Universität Göttingen

M.Hildermeier@phil.uni-goettingen.de

6. L. Häfner, Stadtdumawahlen und soziale Eliten in Kazan' 1870 bis 1913: Zur rechtlichen Lage und politischen Praxis der lokalen Selbstverwaltung, Jahrbücher für Geschichte Osteuropas, 44, 1996, S. 217-252; G. Haussmann, Universität und städtische Gesellschaft in Odessa, 1865-1917. Soziale und nationale Selbstorganisation an der Peripherie des Zarenreiches, Stuttgart, 1998. 